\title{
¿Tiene un sentido el desarrollo local en el contexto metropolitano? Un enfoque europeo
}

Christophe Demazière Universidad de Tours/CESA

\section{Introducción}

Hace dos décadas que el tema del desarrollo local es objeto de un interés creciente en Europa, visible en el contexto de las políticas de desarrollo puestas en práctica en los años ochenta y noventa, o en los cambios en la literatura económica, geográfica, o de ordenamiento y urbanismo. Los numerosos trabajos sobre los temas del desarrollo local permitieron evidenciar algunos "tipos" de espacios locales: distritos industriales, sistemas productivos locales, medios innovadores, espacios tecnopolitanos, etc. (Benko y Lipietz, 1992; Courlet y Soulage, 1994; Pecqueur, 1996; Scott, 1988). Esto permite comparaciones y la elaboración de recomendaciones en materia de política industrial o de ordenamiento del territorio. En un contexto de crisis económica profunda, numerosas instituciones han hecho suyos los nuevos conocimiento de los procesos locales del desarrollo, para preservar y reforzar las ventajas de su territorio. Así, se puede decir que el espacio local, desde unos quince años atrás realmente ha emergido como un ámbito pertinente de intervención hacia el desarrollo.

Economía, Sociedad y Territorio, vol. II, núm. 6, 1999, 213-238. 
Hoy día, la expansión de las estrategias de desarrollo local a lo largo de Europa obliga a su puesta en perspectiva en el conjunto de los procesos generales de desarrollo económico que no son forzosamente -inclusive, no principalmente- "locales". A propósito, Ganne nota irónicamente que "por el lado que se lo vea, en investigación o en política, en el campo de lo rural como con relación a lo urbano, desde un enfoque económico o cultural, en historia, en sociología y hasta en antropología, todo designa lo local como la nueva evidencia, el nuevo campo que se impone" (Ganne, 1985:23). En otros términos, es preciso identificar bien las ventajas y los límites del desarrollo local, según los contextos económicos, geográficos y políticos contemplados.

Por ende, es normal y natural que los resultados de las estrategias de desarrollo local sean examinados de forma crítica, tanto más, dado que otros temas como la metropolización o la globalización pueden aparecer hoy más esenciales a los ojos de numerosos investigadores y tomadores de decisiones para el desarrollo futuro de las ciudades. En Europa, la noción de metropolización remite al hecho de que algunas ciudades de grandes dimensiones conocen desde mediados de los años ochenta, un renacimiento económico y demográfico, mientras que durante los años sesenta y setenta su poder de atracción disminuyó para los hogares y las empresas (Lacour, 1993). La noción de globalización económica es a la vez más difusa y discutida (Sassen, 1996; Shachar, 1997). Para simplificar, puede plantearse que remite esencialmente a tres fenómenos: la emergencia de mercados financieros interconectados, la difusión de nuevas tecnologías de información y la constitución de redes intercontinentales de comunicación, así como la progresión de grandes empresas internacionales en los intercambios internacionales (Demazière y Rodríguez, 1999). Estos cambios económicos tienen implicaciones espaciales y organizacionales para algunas ciudades cuya economía se integra cada vez más en redes transnacionales de producción e intercambio (Sassen, 1996).

Las tesis sobre la metropolización y la globalización tienen en común el argumento según el cual, esencialmente es respecto de las grandes aglomeraciones que se ejercen hoy las lógicas de crecimiento económico y de creación de empleos calificados. Si se lleva al extremo, toda estrategia local de desarrollo en un medio metropolitano se expresará forzosamente en el contexto de una economía internacional de competencia (Amin y Thrift, 1994). Por otra parte, las estrategias de desarrollo local de hecho son 
desempeñadas por agentes sociales extremadamente diversos: gobiernos locales, empresarios e instituciones que los representan, sindicatos de asalariados, movimientos sociales y políticos. Todos estos agentes no manifiestan una misma racionalidad y un mismo objetivo. Existen, por ejemplo, actores locales preocupados no tanto por insertar la economía de una ciudad en la globalización, isino por integrar a las personas en la economía local! Son las iniciativas de desarrollo de estos agentes, portadoras de una mayor justicia social, las que nos interesan en este artículo. Trataremos de mostrar que las iniciativas locales de desarrollo que ocurren en un contexto metropolitano, tienen ciertas ventajas, pero también ciertas limitaciones. Se enfrentan a restricciones a las que las estrategias de desarrollo rural están menos sometidas a priori. En un contexto metropolitano, en el cual se concentran las actividades estratégicas y los capitales internacionales, en el que la población misma parece relativamente móvil, ¿cuáles son las posibilidades objetivas de definir y conducir estrategias locales de desarrollo?

De antemano conviene insistir sobre las diferencias de escala entre los fenómenos de desarrollo local, por una parte, y los procesos de metropolización y globalización, por la otra. Mientras que las iniciativas locales de desarrollo se preocupan, en la mayoría de los casos, por unos miles de personas, la metropolización afecta -selectivamente- concentraciones humanas que suman a cientos de miles; por su parte, la globalización concentra sus efectos en las capitales mundiales "multimillonarias". En el primer caso, la apuesta será frecuentemente la de crear o mantener algunas decenas o centenas de empleos; en el otro, se debe pensar por lo menos en millares de puestos de trabajo. Desde una perspectiva geográfica, en el primer caso se asiste a un regreso al barrio, mientras que en el otro se vislumbra la formación de un archipiélago mundial del desarrollo hecho de "ciudades y vacíos" (Dollfus, 1997). Por una parte, el desarrollo local quiere volver a colocar al ser humano en el centro de los procesos económicos; por la otra, la metropolización y la globalización parecerían ser las expresiones finiseculares del capitalismo.

A pesar de estas diferencias -o más bien, debido a esas diferencias-, nos parece que a veces en el seno de las grandes metrópolis hay lugar para iniciativas locales de desarrollo enfocadas hacia ciertas partes de la aglomeración o ciertos subconjuntos bien identificados de población. La lógica del desarrollo local se justifica, entre otras circunstancias, cuando el ritmo rápido del 
cambio económico se traduce, en ciertos espacios intraurbanos, en numerosos cierres de empresas, aparición de "bolsones de pobreza" o fenómenos de decadencia urbana. El crecimiento económico, así como una vigorosa política de ordenamiento del espacio físico, no son en sí los garantes del desarrollo del conjunto de la población metropolitana (Stevens, 1997).

En un primer momento examinaremos, en el contexto europeo, los objetivos y las finalidades de las estrategias de desarrollo local en el medio urbano. En seguida ilustraremos nuestros propósitos con el estudio de una estrategia de desarrollo innovadora, implantada en un barrio de una gran metrópoli europea, Amberes, segunda ciudad de Bélgica (con 900000 habitantes) y, antes que todo, la segunda metrópoli portuaria de Europa.

\section{El desarrollo local en el medio metropolitano: una expansión reciente en Europa}

El inicio de la crisis de las economías europeas avanzadas a mediados de los años setenta, ha abierto indudablemente, para los actores privados y públicos, una fase de puesta en tela de juicio de sus actividades y de redefinición de sus competencias. Es particularmente el caso respecto al ordenamiento del territorio, en que los sistemas de planificación urbana existentes se han revelado incapaces para responder a dos nuevas apuestas: la reestructuración económica y la revitalización urbana.

De manera general, la búsqueda de un nuevo crecimiento económico por parte de diversos tipos de actores, dio lugar a la implementación de acciones, políticas y estrategias muy diferentes de aquellas definidas en los años sesenta (Albrechts, 1991). De acuerdo con los diversos países, también resultan muy distintas las vías exploradas. Sin embargo, casi todas han sido condicionadas por un principio de autoridad presupuestal adoptado progresivamente por la mayoría de los gobiernos europeos, independientemente de su ideología. Se puede observar que las instituciones supranacionales, entre otras la Unión Europea, han desempeñado un papel creciente, esta última mediante sus fondos estructurales.

Así, se ha asistido a una multiplicación de los esfuerzos en el ámbito local hacia el desarrollo económico y la cohesión social. Esta búsqueda de un desarrollo local dentro de las regiones, de las aglomeraciones, o de las ciudades, inclusive de los barrios, 
a veces ha ido acompañada por una descentralización de los poderes (Moulaert y Demzière, 1996).

\subsection{El cambio de escala de la acción pública}

A lo largo de Europa se asistió a un cambio nítido de escala en la intervención pública. Según Swyngedouw (1999) se percibe a un "vaciamiento" del Estado central por arriba (la Unión Europea) y por abajo (el ámbito regional y local). Articulados con los fondos europeos disponibles, los actores locales, públicos, privados y asociativos, juegan hoy un papel esencial en la estructuración del espacio urbano (Demazière, 1996). La innovación social nace de las asociaciones que surgen entre estos diversos actores.

Con relación al desarrollo económico, los últimos quince años se caracterizan entonces por un multiplicación impresionante de las iniciativas locales. Dado el acento puesto en la responsabilidad, la autonomía y la coordinación, dichas estrategias parecerían romper con las políticas de desarrollo anteriores a la crisis. Durante los años cincuenta y sesenta, la gobernabilidad no se planteaba como una variable importante del desarrollo económico de las regiones y de las ciudades. Se pensaba, por ejemplo, que el desarrollo de las regiones "subindustrializadas" sería la consecuencia de las grandes políticas nacionales de infraestructura y de ordenamiento del territorio (Stöhr y Taylor, 1981; Rocherfort, 1995). Pero el semifracaso de las políticas de ordenamiento del territorio en diversos países europeos, demostró que la concentración de ciertas cantidades de capitales humanos y financieros sobre un espacio determinado, no generaba mecánicamente el desarrollo.

Esta constatación también vale en buena medida para las zonas metropolitanas. En Francia, por ejemplo, las preocupaciones de los gobiernos locales elegidos en las grandes ciudades fueron, durante los "Treinta gloriosos años", centradas exclusivamente sobre las cuestiones de la vivienda, del dominio del mercado inmobiliario, de los transportes y de los equipamientos (Ganne, 1985). Es el Estado central que mantenía el privilegio de impulsar el desarrollo económico de las ciudades y las regiones, entre otros medios, con la política de ordenamiento del territorio. Con la ruptura del modo de crecimiento fordista y las leyes de descentralización de 1982-1983, el tema del desarrollo local emergió progresivamente. Desde una perspectiva crítica, Préteceille (1989) 
nota que en la actualidad se llega a un esquema casi inverso de repartición de las atribuciones entre el Estado central y los gobiernos locales. El Estado se atribuye un papel secundario en la economía, mientras que la política de los gobiernos locales tiene por objeto fundamental el desarrollo económico. La misma evolución puede ser verificada a lo largo de Europa Occidental, con contrastes que se deben, entre otros aspectos, a grados diferenciales de descentralización del aparato político-administrativo y, en particular, de la tutela que a veces han sabido conservar los Estados centrales sobre los poderes locales (véase para el caso de Europa del Sur, Carrière y Demazière, 1998). Los márgenes de maniobra concedidos a las grandes ciudades, así como la experiencia que éstas adquirieron mediante sus políticas económicas y sociales locales, pueden volverse a utilizar en acciones orientadas hacia barrios o poblaciones específicas.

\subsection{La polarización social y espacial, nueva apuesta para las metrópolis europeas}

Se torna cada vez más evidente que las grandes ciudades europeas y las poblaciones que albergan se ven confrontadas a nuevas desigualdades del desarrollo, mientras que permanecen siempre con nosotros las disparidades constituidas durante los "treinta gloriosos años". Ciertamente, vistas en forma global, las ciudades de gran escala conocen un cierto renacimiento económico desde mediados de la década de los ochenta, mientras que durante los años sesenta y setenta su poder de atracción disminuyó para los hogares y las empresas (Lacour, 1993). Sin embargo, a pesar de su éxito económico relativo se asiste también, en las metrópolis europeas, a la exacerbación del desempleo de larga duración, a procesos de exclusión social y a tensiones entre grupos étnicos. En el medio urbano denso de estas ciudades, la reestructuración económica ha significado frecuentemente el cierre de unidades de producción, o eventualmente su relocalización. Por ende, debido a la viscosidad de los mercados del trabajo y de la vivienda se provoca la formación de "bolsones" de pobreza (Demazière, 1999). En paralelo a la desindustrialización, la terciarización de las metrópolis europeas, y particularmente el desarrollo de actividades de servicios avanzados, genera una polari- 
zación socio-espacial de la población ${ }^{1}$. Es la constatación que se puede hacer en Europa en ciudades tan distintas entre sí como París, Londres, Bruselas, Amberes, Estocolmo, Bristol... (Martens y Vervaeke, 1997). En cada caso, el proceso de reestructuración económica discrimina sensiblemente la potencialidad de alcanzar un empleo en función del sexo, la pertenencia étnica, la edad o el grado de capacitación.

Respecto a lo espacial, las nuevas desigualdades económicas y sociales se conjugan para producir una ciudad "estallada" (Haumont y Levy, 1998). Algunos sectores de las grandes aglomeraciones acumulan las desventajas, en términos del ingreso de las poblaciones, de lo confortable de las viviendas o de los tipos de servicios públicos y privados disponibles. Naturalmente, las poblaciones víctimas de la reestructuración económica no habitan todas en zonas urbanas desfavorecidas, y la mayoría de quienes viven en zonas urbanas degradadas se encuentran perfectamente insertos en la economía y la sociedad (Hall, 1981). Sin embargo, varios estudios han puesto en evidencia una cierta dualización del espacio urbano en el caso de ciudades como Bruselas, Amsterdam, Londres o Hamburgo (Kesteloot, 1994; Kloosterman, 1994; Fainstein et al., 1992; Dangschat, 1994).

En las grandes ciudades del norte de Europa dos tipos de espacios intraurbanos se encuentran particularmente privados de desarrollo. Se trata, en primer lugar, de los barrios obreros situados en las zonas pericentrales de las ciudades. Estos barrios fueron creados durante el siglo XIX en el curso del poderoso movimiento de industrialización de Europa Occidental, y están formados, en la mayoría de los casos, por alineamientos de pequeñas viviendas apretadas en torno a fábricas o en las cercanías de instalaciones portuarias. El otro tipo de zona urbana desfavorecida corresponde a los grandes conjuntos de vivienda social erigidos en la periferia de las grandes ciudades. Frecuentemente inspirados por un urbanismo funcional que demostró sus límites, desde hace veinte años estos barrios han conocido importantes movimientos de pérdida de población, y un debilitamiento progresivo de las fuentes locales de empleo (Duprez y Macrakis, 1996). Este tipo de espacio predomina en las políticas hacia la ciudad en Francia. Por el contrario, con menos frecuencia los

\footnotetext{
${ }^{1}$ No trataremos aquí el caso, aún más dramático, de algunas cuencas de antigua tradición industrial, que durante los últimos treinta años han conocido el desplome de sus sectores principales de actividad, la recesión de las industrias asociadas, y un débil crecimiento de las actividades de servicios (Swyngedouw, 1999).
} 
barrios obreros son objeto de rehabilitaciones y resultan menos estudiados por los investigadores. Sin embargo, su estado avanzado de degradación requiere acciones de redesarrollo, particularmente para ofrecer a los habitantes oportunidades de empleo $\mathrm{y}$ condiciones dignas de vivienda.

En este contexto preciso, la noción de desarrollo local ofrece cierta pertinencia. En efecto, un crecimiento económico impulsado en la escala metropolitana no parece capaz de detener el proceso de degradación de estos barrios, que, en la práctica, se encuentran desconectados de los flujos económicos y demográficos por los que atraviesa cualquier metrópoli relevante. Por el contrario, es posible que las iniciativas locales en la escala de estos barrios sean susceptibles de alcanzar efectos significativos para su regeneración.

\subsection{Estrategias de desarrollo local que intentan conciliar la eficacia económica y la justicia social}

El desarrollo local a que nos referimos aquí, empieza por la identificación, por parte de una población local o de sus representantes, de los problemas económicos locales; desemboca luego en la puesta en práctica de iniciativas tendientes, por diversos medios, al mejoramiento de las condiciones económicas y físicas de los grupos sociales llamados desfavorecidos (desempleados, minorías étnicas, jóvenes sin ocupación, etc.). Entre las iniciativas posibles podemos citar:

- El estímulo de los servicios de proximidad en las zonas urbanas de las que ha salido la actividad económica de base.

- El respaldo a los trabajadores independientes y a quienes sustentan proyectos para la capacitación, la puesta a disposición de espacios físicos, la provisión de servicios diversos, o las asesorías.

- La oferta de facilidades crediticias fuera de las estructuras clásicas de financiamiento (microcréditos, banca solidaria).

- Acciones de capacitación orientadas a ciertas categorías de población identificadas, que permiten realizar trabajos de mejoramiento del medio ambiente o de reforzamiento de los lazos sociales. 
Con frecuencia el elemento detonador de este tipo de desarrollo será la identificación de un problema específico, para el cual no se puede encontrar alguna institución establecida en materia de desarrollo urbano. Podemos citar, por ejemplo, la degradación barrial de viviendas individuales privadas, y el costo demasiado elevado de su mantenimiento para los propietarios que las ocupan, frente a su nivel de ingreso. En este caso, la solución puede ser la de instaurar una oficina de asesoría y de préstamo de herramientas, estimulando la reparación de las viviendas por parte de los habitantes. La movilización local sobre este problema restringido puede luego desembocar en un dinámica colectiva con la voluntad para enfrentar asuntos como la desaparición de los comercios, el mal estado de las vías públicas, etcétera.

También en el origen del desarrollo local de barrios desfavorecidos se encuentra la constatación de que las estrategias tradicionales de desarrollo de las ciudades -que buscan, antes que todo, estímulo al aspecto general de actividad de una economía local-, casi no producen efectos benéficos para los grupos sociales menos privilegiados. Existe entonces una posible tensión, por una parte, entre las instituciones encargadas del desarrollo de los barrios y que privilegian el intento de conciliar la eficacia económica y la justicia social, y por otra parte, las instituciones a cargo de la escala metropolitana, del desarrollo económico. En los Estados Unidos esta tensión es particularmente notable: Mientras que las estrategias de las ciudades se limitan tradicionalmente a eliminar las barreras a la inversión privada, por mucho tiempo el desarrollo llamado comunitario ha sido enfrentado por las minorías raciales (Wilson, 1996). En un contexto metropolitano, las iniciativas orientadas hacia el desarrollo de un barrio o población específica, demuestran la particularidad de insertarse en un medio denso en instituciones a cargo del ordenamiento y del desarrollo (desde la cámara de comercio hasta la oficina de urbanismo, pasando por la autoridad electa). Las iniciativas locales de desarrollo de pronto se ven confrontadas, en este caso, a instituciones y procesos que actúan en una escala espacial mayor. Las grandes aglomeraciones europeas no siempre tienen organismos ejecutivos electos para su particular escala, pero con frecuencia sostienen poderosas instituciones de desarrollo económico. En este caso se vislumbra una vasta diversidad. En algunos contextos, puede ocurrir la formación de asociaciones equitativas entre las organizaciones que promueven un desarrollo local y aquellas que actúan a escala de la metropolización y de la globalización. 
La contracción del sector público en diversos países europeos durante los últimos quince años, estimuló la complementariedad de las estrategias de desarrollo local con relación a la intervención pública tradicional, preocupada por la preservación del espacio urbano y/o del desarrollo económico. A veces las autoridades públicas son forzadas a reconocer los límites (entre los cuales están los financieros) de su capacidad de acción, de tal suerte que involucran a las organizaciones de la sociedad civil. Esta orientación se desarrolla, entre otros aspectos, gracias a los programas de acción de la Unión Europea. Ésta desempeñó un papel precursor en los años ochenta mediante los programas Pobreza III y URBAN. Algunas innovaciones institucionales derivadas del programa Pobreza III (llevado a cabo desde 1989 hasta 1993) fueron retomadas en países de tradición social-demócrata, como es el caso de Dinamarca, pero también en países que no conocieron la fase del Estado Benefactor, como en Portugal, o aquellos que lo han revisado considerablemente, como Gran Bretaña. La emergencia de organizaciones sin fines de lucro que emprenden acciones de desarrollo local, representa una ventaja económica para las autoridades locales: estas organizaciones obtienen fondos que resultan inaccesibles para los gobiernos locales. Además su participación es susceptible de mejorar el ambiente local de los negocios (Stoker y Young, 1993).

Aparte de los conflictos o competencias potenciales entre instituciones con visiones y culturas distintas, los límites del desarrollo local en el medio urbano refieren a dos dimensiones. En primer lugar, las estrategias son llevadas cabo en una escala espacial reducida que a lo sumo representa algunas decenas de miles de habitantes. La ampliación de esta escala implica el riesgo de una pérdida de eficacia de las acciones. Para evitar estos obstáculos debe buscarse la reproducción de las experiencias en pequeña escala tomando en cuenta cada vez el contexto específico. En segundo lugar, las organizaciones para el desarrollo local son frecuentemente de pequeña escala, lo que puede conllevar una elevada tasa de fracaso de sus iniciativas socioeconómicas. Lo anterior puede acompañarse de una intensa dependencia financiera con relación a las instancias públicas locales, regionales, nacionales o europeas. En este caso, la obtención de financiamiento puede ser considerada como prioritaria antes que la definición de acciones que concilian la eficacia económica con la justicia social; así mismo, la dependencia con relación a los fondos públi- 
cos puede generar problemas en caso de un cambio de orientación de los poderes públicos (MacFarlane, 1993; Wilson, 1996).

Finalmente, los dos rasgos originales del desarrollo local en el medio metropolitano, tal como se practica en Europa, son, por una parte, un enfoque multidimensional de la crisis y sus efectos sobre las poblaciones urbanas; por la otra, la promoción de soluciones "desde abajo". Es interesante observar cómo las instituciones que se involucran en este desarrollo local, interactúan con los actores formales del desarrollo de las ciudades (empresas, autoridades locales, regionales y nacionales, cámaras de comercio, etc.). En el caso de Quebec, D. G. Tremblay (1996) apunta que las organizaciones del desarrollo llamado comunitario, a pesar de sus resultados favorables, se ven forzadas a demostrar constantemente que sus acciones constituyen soluciones eficaces a los problemas de desindustrialización, de exclusión social o de degradación del medio ambiente urbano. En aquellos lugares en que el movimiento relacionado con el desarrollo local es más reciente y menos estructurado, como en el caso europeo, se plantea con insistencia aun mayor el tema de la justificación del modelo de acción que representa. Sin embargo, el conocimiento de experiencias de estrategias innovadoras y que han producido importantes resultados en materia de renovación urbana, podría favorecer el auge del desarrollo local en el medio urbano. Desde esa perspectiva, la estrategia puesta en práctica en el barrio nordeste de Amberes desde hace más de diez años presenta cierto interés.

\section{Una estrategia de desarrollo local innovadora: El caso de Amberes}

La segunda comuna de Bélgica (con 500000 habitantes); centro de una región urbana que está próxima al millón de habitantes; el segundo puerto de Europa Occidental por sus intercambios; Amberes es una ciudad cuyo desarrollo económico está íntimamente ligado a la historia del continente europeo. En el siglo XVI, casi al inicio de los grandes descubrimientos continentales, según las palabras de Fernand Braudel, Amberes era "la capital económica de Europa, es decir, del mundo" (Braudel, 1979:118). Entre 1850 y 1914, lapso de industrialización de Europa Occidental, el puerto de Amberes, bien situado y bien equipado, veía multiplicarse por sesenta su tráfico. Entonces la ciudad conoció 
la cuatriplicación de su población, haciendo necesaria la creación de barrios destinados a la vivienda de la mano de obra portuaria. Entre 1920 y 1975 el puerto y la región urbana de Amberes conocieron una vasta industrialización, principalmente establecimientos de filiales de empresas multinacionales (como GM, Ford, BASF, Bayer, Exxon, Monsato, etc.), en la industria química y petroquímica, automotriz o farmacéutica (Demazière, 1999). Verdadero concentrado del fordismo, Amberes conoció desde hace 25 años un cambio económico rápido, que refuerza las desigualdades del desarrollo en el seno de la aglomeración, planteando así el tema de las estrategias locales.

\subsection{Los efectos del cambio económico rápido en Amberes: una polarización socio-espacial en crecimiento}

En una primera impresión, la situación económica de Amberes parece notoria. Entre 1972 y 1992 el empleo asalariado total en el distrito pasó de 334000 a 353000 unidades, esto es, una alza de 5.7\%; en el mismo lapso retrocedió 7\% en Bélgica. Por el contrario, la componente industrial retrocedió sensiblemente en la situación de Amberes. El sector secundario representaba 153 mil empleos en 1972 y solamente 108000 veinte años después, o sea, un retroceso de $28.9 \%$. El crecimiento del sector terciario (de 180000 empleos en 1972 a 244000 en 1992) esconde dicho fenómeno. De hecho, la crisis económica mundial provocó una fuerte caída del empleo industrial en todas sus actividades. Sólo el sector fuertemente capitalizado de la química se reporta como una excepción, quizá debido a la posición geoeconómica de privilegio del puerto de Amberes.

Hoy como ayer, el puerto es el motor del crecimiento de Bélgica. En 1994 el conjunto de las actividades que tenían lugar en el puerto de Amberes (distribución y almacenamiento, producción industrial, servicios privados, sector público) contribuía con 3.5\% del PNB de Bélgica, es decir, un porcentaje mayor que el de la contribución nacional de la agricultura o la industria textil. Ciertamente el tráfico portuario ha conocido una reducción severa en el momento de la primera crisis petrolera, pero posteriormente recobró su crecimiento hasta alcanzar 110 millones de toneladas en 1994. El objetivo de las autoridades locales es que el tráfico portuario alcance 150 millones de toneladas en el año 2010. Con esta finalidad se han realizado importantes inversio- 
nes públicas; $50 \%$ han sido financiadas por las autoridades portuarias (la ciudad de Amberes) y por la Región Flamenca, que tomó el relevo del Estado belga en el marco del proceso de federalización del país. No obstante, resulta claro que el desarrollo inducido por las grandes inversiones aporta un contenido en empleos netamente menor que en el pasado (Demazière, 1999). Una gran parte de las actividades de descarga y de almacenamiento alcanzan un carácter semiindustrial, y a pesar del hecho de que el tráfico portuario no ha dejado de incrementarse, el contingente de estibadores no cesa de reducirse. Así mismo, el empleo en la industria portuaria disminuyó $10.5 \%$ entre 1984 y 1992 en términos globales, pasando de 36000 a 32000 personas (Adriaenssens y Geldof, 1997). La desconexión entre el empleo y las inversiones industriales en parte es provocada por el origen de la inversión. Diez empresas, que de hecho son filiales de multinacionales, aportan la mitad del valor agregado de las inversiones y del empleo localizados en el puerto. Por otra parte, entre todas las empresas, son las multinacionales las que han emprendido en forma más sistemática políticas de racionalización (Dicken, 1992). Además, por realizar su reestructuración en el ámbito mundial, con frecuencia han modificado tanto la inserción de sus filiales en la economía internacional, como la relación de éstas con su entorno local.

El cambio rápido en la estructura del empleo en Amberes tiene como contraparte una diferenciación intrarregional relevante. En un estudio algo antiguo, B. De Borger y D. Van der Wee (1986) demostraron que el municipio de Amberes perdió globalmente, entre 1974 y 1984, más de 20000 empleos, mientras que la periferia ganó 5 000; ellos destacan que el empleo industrial retrocedió 19000 unidades en la zona central, mientras que la periferia sólo perdió 3000 empleos en ese sector; finalmente, destacan que el crecimiento del terciario tuvo sobre todo su verificación en la periferia con una ganancia de 10000 empleos, frente a los 5000 del municipio de Amberes. Lo anterior se ha visto confirmado por la evolución del desempleo en Amberes. A pesar de un relativo crecimiento económico de la región urbana, de una importante expansión de las actividades portuarias -no obstante las supresiones de empleos- y de un crecimiento del empleo en los servicios, el desempleo aumentó en la aglomeración de Amberes a fines de los años ochenta (Adriaenssens y Geldof, 1997). La mano de obra industrial de cierta edad, los jóvenes y los inmigrantes sin calificación, son los sectores más 
afectados por el desempleo de larga duración. Cabe recordar que una proporción importante de estas poblaciones se reagrupa en el corazón de la región urbana, y particularmente en los barrios populares construidos en el siglo XIX, en la época de la edad de oro del puerto de Amberes.

\section{Cuadro 1}

La evolución del ingreso medio por habitante en el municipio de Amberes, en su distrito y en Bélgica, 1970-1992 (en francos belgas a precios corrientes)

\begin{tabular}{|lccc|}
\hline Año/Lugar & 1971 & 1981 & 1990 \\
\hline Municipio de Amberes & 75000 & 213000 & 323500 \\
Distrito & 67600 & 214000 & 335400 \\
Bélgica & 57200 & 191800 & 300000 \\
\hline
\end{tabular}

Fuente: Instituto Nacional de Estadística de Bélgica.

La polarización socio-espacial de Amberes también puede medirse por medio de la evolución del ingreso de los hogares (cuadro 1). Durante los últimos veinte años, éste se ha incrementado en forma regular en el municipio y el distrito de Amberes, manteniéndose permanentemente más elevado que el ingreso por habitante en Bélgica. Sin embargo, se constata que el ingreso per cápita en el municipio se ha vuelto inferior al correspondiente en el distrito de Amberes, mientras que ocurría lo contrario al inicio de los años setenta. Este proceso se relaciona con la expansión de la suburbanización residencial iniciada en los años sesenta. De Brabander et al. (1992) han demostrado que más bien son los miembros de los hogares más favorecidos quienes dejan el municipio, mientras que, en forma simétrica, los grupos sociales menos favorecidos llegan o se mantienen en la ciudad.

\subsection{La estrategia del desarrollo local del nordeste de Amberes}

El nordeste de Amberes constituye un barrio de 33000 habitantes, situado en las inmediaciones del rico centro histórico de la ciudad. Construido durante la segunda mitad del siglo XIX, este barrio obrero se encuentra involucrado desde hace varias décadas en un grave retroceso económico, social y del entorno físico. El medio construido, esencialmente privado, es antiguo, poco 
confortable y escasamente renovado o transformado. El nordeste de Amberes tiende a concentrar una población desfavorecida, a veces de origen extranjero. Por otra parte, debido al reducido crecimiento de los ingresos, la oferta local de servicios personales se ha debilitado bastante durante los últimos quince años.

Esta degradación urbana se inicia en la edad de oro del crecimiento económico de Bélgica (los años cincuenta y sesenta), pero la crisis económica de los últimos veinte años ha acelerado el proceso. En el contexto de la reestructuración económica, social y espacial de la región urbana de Amberes durante los años setenta y ochenta, los desfases entre el barrio y el resto de la aglomeración se han acentuado. Por una parte, la dinámica del mercado laboral desfavorece a las poblaciones con escasa capacitación, al destruir los empleos de poca o nula calificación. Por otra parte, el pequeño número de viviendas de interés social en Amberes, conduce a una concentración de categorías de población poco desfavorecidas, en las viviendas remanentes privadas del barrio. Éste cuenta en la actualidad con más desempleados en términos relativos y menos diplomados que el resto de la municipalidad y que la región urbana. La proporción de población extranjera en el barrio ha pasado de 5\% en 1970 a 28\% en 1995 (para la municipalidad de Amberes, las cifras son de 8 y $12 \%$ respectivamente).

Un "Atlas de la pobreza en Amberes", elaborado en la segunda mitad de los años ochenta, demostró que el barrio Nordeste de Amberes se constituye en un espacio desfavorecido (Marynissen et al., 1987 y 1988). Cuando se publicó, varias instituciones percibieron la necesidad de plantear un nuevo enfoque para contrarrestar la dualización en curso del espacio urbano y la exclusión de algunas categorías sociales. En este contexto, siete instituciones asociadas -entre las que se cuenta la ciudad de Amberes, la Fundación Rey Balduino, la Institución Regional de Trabajo Social y la Universidad-crearon en abril de 1990 la Sociedad de Desarrollo del Barrio Nordeste de Amberes (conocida como вом). ${ }^{2}$ Esta nueva organización tenía por objetivo el de poner en práctica un enfoque socioeconómico del desarrollo sobre un territorio de dimensión significativa, en el cual pueda coordinarse la intervención de varias instituciones.

La creación de la вом salía un poco del contexto general de las políticas públicas en Bélgica. Ciertamente, desde mediados de

${ }^{2}$ вом corresponde a las iniciales del nombre de la estructura en flamenco Buurtontwikkelingmaatschappij Noord-Oost Antwerpen. 
los años setenta las autoridades públicas belgas -en los ámbitos local, regional y nacional- se han preocupado por la reaparición de pobreza en Bélgica. Unos centros municipales de intervención social proveen ciertas prestaciones a las familias y a las personas, entre otras, un ingreso mínimo; por otra parte, diversas asociaciones emprenden acciones directas para reforzar las redes sociales en los barrios desfavorecidos. Las políticas de vivienda y de ordenamiento urbano han propuesto algunas medidas para las zonas desfavorecidas, pero sus efectos han sido débiles. El espacio público pudo mejorarse, pero pocas viviendas han sido renovadas, a pesar de los subsidios otorgados a los propietarios privados. Con relación a la política de empleo, sólo una pequeña fracción de los fondos disponibles se orienta a la capacitación y a la reintegración de las categorías sociales excluidas.

La creación de la BOM constituyó una innovación con relación a los enfoques tradicionales acerca de los problemas económicos y sociales en las ciudades flamencas, que se centraban en los aspectos sociales o socioculturales en una escala espacial extremadamente reducida. Durante sus cuatro primeros años de existencia, la вом ha sido financiada por la Unión Europea en el marco de su tercer programa para la integración de los grupos menos favorecidos (Pobreza III). La acción de la вом fue posteriormente reconocida como "proyecto piloto urbano" de la Dirección General XVI de la Unión Europea. Actualmente, la вом ha sido integrada en el programa europeo Urban, que apoya sus proyectos económicos, mientras que sus iniciativas sociales más bien se financian con los fondos para la integración social ofrecidos por el gobierno regional flamenco.

Las diversas iniciativas de la вом se despliegan en tres direcciones: la intervención económica, sobre la vivienda, y la animación sociocultural (cuadro 2). Por razones de comodidad, hemos clasificado estas acciones en función del eje principal de desarrollo al cual se refieren. Sin embargo, se debe recordar que numerosos proyectos, si no todos, son pluridimensionales. Tomemos el ejemplo de la sala de deportes construida básicamente por jóvenes que han abandonado los estudios. En este caso un sistema de capacitación que incluye la "formación y el trabajo" ha sido diseñado especialmente, lo que implicó que la escuela profesional asociada a este proyecto tuviera que modificar los contenidos y la forma de su enseñanza. Los jóvenes involucrados han descubierto por primera vez el mundo profesional, tomado confianza en sus capacidades, medido los esfuerzos necesarios 


\section{Cuadro 2 \\ Las principales realizaciones de la вом}

\begin{tabular}{|ll|}
\hline Campos de intervención & Principales proyectos realizados \\
\hline Economía & - Centro de recepción y de orientación para los \\
& buscadores de empleo (Werkwijzer). \\
& -Centro de formación a las nuevas tecnologías \\
& (ATec). \\
& - Incubadora de empresas, reconocida por las \\
& autoridades regionales y proveedora de locales \\
& y servicios a personas con proyectos empresa- \\
& riales de escala humana (NOA Business Center). \\
Vivienda & - Centro de asesoramiento financiero y técnico \\
& para la renovación habitacional (Woonwijzer). \\
& - Rehabilitación de microterritorios, combinan- \\
& do la renovación de las viviendas, el reordena- \\
& miento del espacio público, y el interés en de- \\
& sarrollar las redes sociales de vecindario. \\
& - Rehabilitación de viviendas sociales, permi- \\
& tiendo también la reinserción alejada del mun- \\
& do laboral. \\
& - Creación de una sala de deportes destinada a \\
los jóvenes del barrio y construida por jóvenes \\
bajo contratos de formación laboral. \\
- Edición de un periódico trimestral del barrio, \\
con tiraje de 22,000 ejemplares, distribuido gra- \\
tuitamente, redactado por los habitantes, y par- \\
cialmente financiado por inserciones publicitarias. \\
- Guía anual de las asociaciones y organizaciones \\
sociales y culturales del barrio, totalmente auto- \\
financiada. \\
\\
\end{tabular}

para encontrar un trabajo posterior, y cobrado un pequeño salario. Además, la sala de deportes costó la mitad de lo que habría costado si hubiera sido construida y arreglada totalmente por una empresa particular. Por otra parte, su apertura respondió a una necesidad social importante, ya que ninguna sala pública resultaba accesible para los jóvenes de este barrio de 35000 habitantes. Finalmente, son varias asociaciones de jóvenes las que administran esta sala, lo que debería desarrollar su sentido de las responsabilidades y derrumbar muchas barreras originadas en prejuicios raciales.

Aun cuando el ámbito sociocultural mantenga numerosas relaciones con la economía y la vivienda, nos centraremos ahora 
en las acciones llevadas a cabo por la вом en estos dos sectores. Se tratará de evaluar los efectos de las acciones de la BOM sobre los dos problemas más importantes del barrio: el empleo y la vivienda. Se harán evidentes las ventajas de las iniciativas locales de desarrollo, con relación a las políticas públicas tradicionales o a las acciones de desarrollo "desde arriba", incluso cuando se presenten ciertas limitantes.

\subsection{Evaluación de las iniciativas llevadas a cabo}

En el ámbito de la vivienda, el programa más importante de la BOM ha sido la creación de una oficina de asesoría sobre la vivienda: Woonwijzer, que es antes que todo un sitio de información y de asesoramiento para los vecinos del barrio, en materia de renovación y reconversión de su vivienda. En particular, una de las tareas del Woonwijzer es la de difundir las informaciones sobre las disposiciones públicas de ayuda a la renovación (entre otras, los subsidios acordados por el gobierno regional flamenco). El Woonwijzer ayuda a los candidatos a llenar los formularios para lograr un subsidio, y les asiste en su relación con la administración.

Woonwijzer se plantea también ofrecer un asesoramiento en cuanto a la concepción de los planos individuales de renovación. Apoya también la estimación del costo total de la misma. Además, unos especialistas pueden ayudar a las personas en cuanto a las prioridades en la renovación (por ejemplo, la reparación de los techos debe preceder a la modernización del circuito eléctrico, que a su vez tendrá lugar antes que la decoración de los cuartos). Dejadas a su propia iniciativa las familias no harían forzosamente las reflexiones más pensadas, lo que podría traducirse en un uso erróneo de sus recursos. Por último, Woonwijzer también presta herramientas a los habitantes, entre otras un gran andamio que puede ser entregado y armado en el lugar de la obra. Si un cliente viene a pedir prestadas más herramientas de lo necesario, el personal de Woonwijzer examina con él si requiere consejos específicos o apoyo material.

Durante sus dos primeros años de existencia, Woonwijzer ha atendido a 700 familias. Esto constituye un éxito, aun si existen 22000 hogares en el barrio Nordeste de Amberes, de los cuales sólo 6000 son dueños de sus viviendas. Las familias de referencia son jóvenes; en dos tercios de los casos el jefe de fami- 
lia tiene menos de 40 años. La mayoría dispone de un solo salario (50\% de los expedientes), mientras 29\% de los casos tienen dos salarios. Aquella parte de la población para la cual las transferencias sociales (jubilación, seguro de desempleo, ingreso mínimo, etc.) ocupan un sitio importante en el presupuesto familiar, se ha visto poco involucrada en el proceso. Para estas categorías sociales el problema del mejoramiento de las condiciones de vivienda queda aún sin solución. Normalmente, es el sector de la vivienda social el que debería hacerse cargo de esos casos, pero como éste se encuentra poco desarrollado en Flandes, la demanda de vivienda barata y con estándares mínimos de comodidad está poco atendida, pues estos hogares no constituyen una demanda solvente.

En el ámbito de la inserción profesional podemos citar dos acciones de la вом: la creación de un centro de capacitación profesional que desarrolla la innovación tecnológica, social y organizacional (ATec); y la apertura de un centro de recepción y orientación para los demandantes de empleo (Werkwijzer). Estas dos instituciones son originales respecto a los mecanismos clásicos de capacitación y de apoyo al empleo. Desarrollando acciones de capacitación destinadas a personas de escasa formación, desempleadas de larga duración o beneficiarias de un ingreso mínimo, ATec se distingue de los otros organismos de capacitación profesional presentes en Amberes. Éstos sostienen exigencias relativamente elevadas con relación al perfil de los candidatos a los programas de capacitación. Es, entre otros, el caso de VDAB, que es también el organismo de colocación de los desempleados y que maneja procedimientos extremadamente formales y rígidos de selección y de capacitación. ATec se ubicó en un segmento relativamente inferior del mercado laboral, y no pretende evolucionar hacia un segmento superior.

La formación principal ofrecida por ATec es la de técnico en informática. Dura cinco meses e incluye un periodo de estancia en empresa durante cinco semanas. La formación es muy concreta y muy inductiva. Los estudiantes deben resolver casos prácticos de instalación, mantenimiento y reparación de computadoras y de redes. De tal modo que se ven confrontados con problemas comparables con aquellos que pueden planteárseles en una empresa convencional.

ATec es un éxito por lo que concierne a la reinserción en el mercado laboral de personas alejadas del mismo, y con relación a la difusión de una cultura informática en el seno de la población 
del barrio. Son tres las razones por las que este organismo de capacitación es posiblemente único en su género en Amberes. En primer lugar, ATec propone programas de capacitación a personas con un difícil acceso no sólo al empleo, sino también a las formaciones ocupacionales clásicas. En segundo lugar, las tasas de inserción en el empleo son relativamente elevadas. Sin embargo, el hecho de que Amberes sea una economía próspera debe ser tomado en cuenta, pero por otra parte, la atención que ATec presta a las evoluciones del mercado laboral y a la búsqueda de "nichos", es un factor importante para su éxito. Finalmente, la autoevaluación de las acciones de capacitación se realiza en forma permanente y sin concesiones, como lo muestra la suspensión temporal de un curso que ha sido juzgado como mal concebido, o también la actitud receptiva respecto a los requerimientos de las empresas.

El mercado de trabajo en Amberes continúa creando empleos relativamente poco calificados. Sin embargo, el crecimiento económico local, si bien alcanza efectos mecánicos para sacar del desempleo a personas factibles de contratarse, tiene pocos efectos sobre aquellas que reúnen un menor grado de formación, un desempleo de larga duración y un origen extranjero. Por una parte, las personas de quienes hablamos están relativamente alejadas del mundo laboral. Por otra, los empleadores frecuentemente tienen reservas para reclutar a tales personas porque suponen que su productividad es menor, amén de otros prejuicios. Por fin, los diversos programas del servicio público del empleo tienen, según uno de los responsables de la BOM, sobre todo efectos favorables para las personas que podrían integrarse por sí mismas a los mercados de trabajo, en tanto que aquellas que forman las categorías en situación de exclusión, sólo son muy poco alcanzadas por estos programas. Lo anterior resalta el interés de las realizaciones de la вом o de las iniciativas de la cámara de comercio. Sin embargo, tomando en cuenta el incremento y la persistencia del desempleo en el nordeste de Amberes desde hace 15 años, en la actualidad lo más difícil es expandir los primeros resultados benéficos, conservando al mismo tiempo la reconocida eficacia de las iniciativas. Entre otros, es necesario tener la capacidad de actuar sobre la oferta de empleo, cuya característica es específica de aquel barrio, así como sobre la demanda que, por su parte, se sitúa en la escala de la región urbana. La formación de asociaciones entre la вом e instituciones que operan en 
ámbitos espaciales más amplios se antoja, entonces, indispensable.

En materia de creación o atracción de actividades económicas, las iniciativas de la вом han hallado profundas dificultades. El caso de la "incubadora" de empresas es significativo de ello. El concepto de centro de empresa o de incubadora apareció en las esferas a cargo del desarrollo regional de Flandes en los años ochenta. Para promover el desarrollo endógeno de regiones "con retraso en su desarrollo", una veintena de incubadoras fueron creadas bajo la autoridad de las sociedades de desarrollo de las provincias (GOM). La BOM tuvo que convencer a la sociedad de desarrollo de la provincia de Amberes y a los medios empresariales, del interés por implantar una incubadora en el medio urbano. Lo anterior no ha sido fácil y el proyecto ha conocido varias evoluciones sucesivas.

$\mathrm{Al}$ inicio la idea era favorecer la creación de actividades económicas por parte de los residentes del barrio; entre otros, los demandantes de empleo. Sin embargo, frente a la desilusión de varios proyectos de economía social, la Bom decidió gradualmente orientarse hacia el sector económico competitivo. Durante un lapso se trató de seleccionar unos proyectos que tendrían efectos inmediatos para el barrio, por medio de la naturaleza misma de la actividad (como la renovación de edificios), o porque generarían servicios atendidos por personas en proceso de reinserción laboral. Esta presencia de objetivos socioeconómicos, aún atenuada, fue empero mal recibida por los interlocutores de la вом, entre otros por el sector empresarial. Es por ello que se decidió que la incubadora operase completamente en el contexto de la economía formal, mientras que la вом desarrollaba proyectos socioeconómicos en otro marco.

En esta nueva versión, la incubadora trata entonces de atraer al barrio unas pequeñas y medianas empresas en creación, relacionadas con cuatro sectores de actividad: los servicios administrativos, los servicios profesionales, la artesanía y la distribución. Se ofrecen en renta, a una tarifa preferencial, unos locales de pequeña dimensión, así como salas de reunión, con capacidad para unas treinta empresas. La incubadora propone también un conjunto integrado de servicios profesionales: gestión, contabilidad, secretariado y mercadotecnia. Dichos servicios también son accesibles para las empresas establecidas en el barrio. La incubadora se ubica en una antigua escuela totalmente rehabilitada; incluye también un restaurante y un espacio para exposiciones abier- 
to a la población local. En total, la incubadora es un éxito con 22 empresas acogidas desde los dos primeros años, lo que representa alrededor de 120 empleos suplementarios para el barrio.

\section{Conclusión}

La mayoría de las grandes ciudades europeas se encuentran actualmente en una fase de transición posindustrial. El rápido cambio económico en estas ciudades, su metropolización o incluso su globalización, puede hacerlas beneficiarias de la creación de riqueza y de empleos, pero produce también decaimiento urbano y el alejamiento de algunas categorías de población con respecto al mercado laboral. En este contexto, algunas iniciativas locales de desarrollo, portadoras de solidaridad y de innovación social, enfrentan los problemas de empleo, capacitación y vivienda, para provocar la emergencia de actividades económicas viables.

En el caso de Amberes, gran metrópoli cuya economía se encuentra ampliamente abierta al mundo, resulta interesante que haya podido emprenderse una estrategia de desarrollo "desde abajo". Produce resultados significativos en términos de inserción laboral, de renovación de la vivienda y de regeneración económica. En estos tres ámbitos, los diversos proyectos llevados a cabo se presentan como complementarios de los mecanismos tradicionales puestos en práctica en otras escalas espaciales, y que alcanzan pocos éxitos para el dinamismo económico y social del barrio Nordeste de Amberes. Por otra parte, la estrategia de la вом, gracias a su carácter multidimensional, constituye una alternati$v a$ a muchas estrategias de desarrollo urbano. El periodo de observación de la actuación de la вом es aún demasiado corto como para percibir todas sus facetas, pero hoy la вом ha sido reconocida en Bélgica como un modelo de acción descentralizada e integrada. Otras instituciones de la misma índole se encuentran en curso de creación en otros barrios de Amberes, o ya están operando en otras ciudades belgas, como Gante. Ciertamente, resultaría interesante estudiar la transferencia de este modelo a un contexto institucional y económico distinto al de Flandes. En el norte de Francia (entre otros sitios, en Lille, Roubaix o Tourcoing) algunos barrios obreros en decadencia podrían ser el marco de este tipo de intervenciones. Desde hace casi diez años estos barrios han conocido a la vez el redesarrollo físico masivo (por la implantación de edificios universitarios o de centros terciarios de 
los que las autoridades locales esperan la reconversión económica del territorio), y en forma independiente el desarrollo de acciones de lucha contra la exclusión. No obstante, ni las inversiones en infraestructura ni los gastos sociales aparecen como capaces de detener la desintegración de estos barrios, y de impulsar un nuevo desarrollo económico con cohesión social. El ejemplo del barrio Nordeste de Amberes demuestra que otras estrategias podrían ser puestas en operación.

Traducción realizada por Daniel Hiernaux y revisada por Christophe Demazière

\section{Bibliografía}

Adriaenssens, S. y D. Geldof (1997), "La polarisation sociale et spatiale d'Anvers", en A. Martens y M. Vervaeke (eds.), La polarisation sociale des villes européennes, París, Anthropos.

Albrechts, L. (1991), "Changing roles and positions of planners", Urban Studies, vol. 28, pp. 123-137.

Amin, A. y N. Thrift (1994), "Living in the Global", en A. Amin y N. Thrift (eds.), Globalization, Institutions and Regional development in Europe, Oxford, Oxford University Press.

Benko, G. y A. Lipietz (eds.) (1994), Las regiones que ganan. Distritos y redes. Los nuevos paradigmas de la geografía económica, Valencia, Edicions Alfons El Magnànim.

Braudel, F. (1979), Civilisation matérielle, économie et capitalisme, xve-XviIIe siècle, Tome 2: Les jeux de l'échange, París, Armand Colin.

Carrière, J.-P. y C. Demazière (1998), "Les disparités spatiales en Europe du sud sont-elles liées au niveau de décentralisation?", Coloquio de GRERBAM: Les Échelles Territoriales du Partenariat Euro-Méditerranéen, Vérone, 12-13 de septiembre. 
Courlet, C. y B. Soulage (eds.) (1994), Industrie, territoire et politiques publiques, París, L'Harmattan.

Dangschat, J. S. (1994), "Concentration of poverty in the landscapes of "booming» Hamburg: the creation of a new urban underclass", Urban Studies, vol. 31, pp. 1133-1147.

De Borger, B. y D. Van der Wee (1986), Recent Evolution of the Antwerp Economy: Structural Changes and Urban Policy, Amberes, SESO-UFSIA.

De Brabander, G., L. Vervoort y F. Witlox (1992), Metropolis: over mensen, steden en centen, Lovaina, Kritak.

Demazière, C. (ed.) (1996), Du local au global. Les initiatives locales pour le développement économique en Europe et en Amérique, París, L'Harmattan.

- (1999), Développement économique et espace urbain, París, Anthropos-Economica.

— y A. Rodríguez (1998), “Globalisation économique et stratégies de développement des villes”, Espaces et Sociétés, núm. 95, pp. 147-160.

Dicken, P. (1992), Global Shift, Londres, Paul Chapman, segunda edición.

Dollfus, O. (1997), La mondialisation, París, Presses de Sciences Po.

Duprez, D. y B. Macrakis (eds.) (1996), "Vivre dans les quartiers «sensibles»", Les dossiers de Profils, núm. 41, Lille, INSEE.

Fainstein, S., I. Gordon y M. Harloe (eds.) (1992), Divided Cities, Oxford, Basil Blackwell.

Ganne, B. (1985), "Du notable au local. Transformations d'un modèle politique", Annales de la Recherche Urbaine, núm. 28, pp. 23-32. 
Hall, P. (ed.) (1981), The Inner City in Context, Londres, Heinemann.

Haumont, N. y J.-P. Lévy (eds.) (1998), La ville éclatée: quartiers et peuplements, París, L'Harmattan.

Kesteloot, C. (1994), "Three levels of socio-spatial polarization in Brussels”, Built Environment, vol. 20, pp. 204-217.

Kloosterman, R. (1994), "Amsterdamned: the rise of unemployment in Amsterdam in the 1980s", Urban Studies, vol. 31, pp. $1325-1344$.

Lacour, C. (1993), “Métropolisation”, en Plan Urbain (ed.), Mutations économiques et urbanisation, París, La Documentation Française.

MacFarlane, R. (1993), "Community Based Economic Development: The British Experience", en D. Fasenfest (ed.), Community Economic Development. Policy Formation in the us and the UK, Londres, Macmillan.

Martens, A. y M. Vervaeke (eds.) (1997), La polarisation sociale des villes européennes, París, Anthropos-Economica.

Marynissen, R., E. Poppe, T. Jacobs y E. Van Hove (1987), Kansarmoede in de Grootstad Antwerpen. Deel I: De Kwaliteit van het Wonen, Amberes, UIA-Fondation Roi Baudouin.

- (1988), Kansarmoede in de Grootstad Antwerpen. Deel II: De Bewoners, Amberes, UIA-Fondation Roi Baudouin.

Moulaert, F. y C. Demazière (1996), "Le développement local dans une époque post-fordiste", en C. Demazière (ed.), $\mathrm{Du}$ local au global. Les initiatives locales pour le développement économique en Europe et en Amérique, París, L'Harmattan.

Pecqueur, B. (ed.) (1996), Dynamiques territoriales et mutations économiques, París, L'Harmattan. 
Préteceille, E. (1989), "Paradoxes politiques des restructurations urbaines. Globalisation de l'économie et localisation du politique", Espaces et Sociétés, núm. 59, pp. 5-26.

Rochefort, M. (1995), Dynamique de l'espace français et aménagement du territoire, París, L'Harmattan.

Sassen, S. (1996), La ville globale, París, Descartes.

Scott, A. J. (1988), New Industrial Spaces, Londres, Pion.

Shachar, A. (1997), "Economic globalization and urban dynamics”, en F. Moulaert y A. J. Scott (eds.), Cities, Enterprises and Society on the Eve of the XXIst Century, Londres, Pinter.

Stevens, J.-F. (1997), "Dialectique en ville: Enjeux urbains et antagonismes économiques de l'exclusion”, Revue d’Économie Régionale et Urbaine, núm. 5, pp. 723-736.

Stöhr, W. y D. Taylor (eds.) (1981), Development from Above or Below?, Londres, John Wiley and Sons Ltd.

Stoker, G. y S. Young (1993), Cities in the 1990s, Harlow, Longman.

Swyngedouw, E. (1999), "La reconversion du bassin minier belge et la restructuration de l'Etat: la démocratie post-fordiste déficitaire", en G. Benko y A. Lipietz (eds.), La richesse des régions, París, Presses Universitaires de France.

Tremblay, D.-G. (1996), "Stratégies de développement économique local: l'expérience de Montréal et du Québec”, en C. Demazière (ed.), Du local au global. Les initiatives locales pour le développement économique en Europe et en Amérique, París, L'Harmattan.

Wilson, P. A. (1996), "Le développement économique communautaire aux États-Unis et en Amérique Latine”, en C. Demazière (ed.), Du local au global. Les initiatives locales pour le développement économique en Europe et en Amérique, París, L'Harmattan. 